\title{
New onset of loss of smell or taste in household contacts of home-isolated SARS-CoV-2-positive subjects
}

\author{
Paolo Boscolo-Rizzo' ${ }^{1}\left[\right.$ Daniele Borsetto $^{2}$ (]) . Giacomo Spinato ${ }^{1}$. Cristoforo Fabbris ${ }^{1}$. Anna Menegaldo ${ }^{1}$. \\ Piergiorgio Gaudioso ${ }^{1} \cdot$ Piero Nicolai $^{3} \cdot$ Giancarlo Tirelli $^{4} \cdot$ Maria Cristina Da Mosto $^{1} \cdot$ Roberto Rigoli $^{5} \cdot$ Jerry Polesel $^{6}$. \\ Claire Hopkins ${ }^{2}$
}

Received: 30 April 2020 / Accepted: 16 May 2020 / Published online: 24 May 2020

(c) Springer-Verlag GmbH Germany, part of Springer Nature 2020

\begin{abstract}
Purpose To estimate the prevalence of smell or taste impairment in household contacts of mildly symptomatic home-isolated SARS-CoV-2-positive patients.

Methods Cross-sectional study based on ad hoc questions.

Results Of 214 mildly symptomatic COVID-19 patients managed at home under self-isolation, 179 reported to have at least one household contact, with the total number of no study participants contacts being 296. Among 175 household contacts not tested for SARS-CoV-2 infection, 67 (38.3\%) had SARS-CoV-2 compatible symptoms, 39 (22.3\%) had loss of smell or taste with $7(4.0 \%)$ having loss of smell or taste in the absence of other symptoms. The prevalence of smell or taste impairment was $1.5 \%$ in patients tested negative compared to $63.0 \%$ of those tested positive for SARS-CoV-2 $(p<0.001)$.

Conclusion Smell or taste impairment are quite common in not-tested household contacts of mildly symptomatic homeisolated SARS-CoV-2-positive patients. This should be taken into account when estimating the burden of loss of sense of smell and taste during COVID-19 pandemic, and further highlights the value of loss of sense of smell and taste as a marker of infection.
\end{abstract}

Keywords COVID-19 $\cdot$ Coronavirus $\cdot$ Anosmia $\cdot$ Smell and taste loss $\cdot$ Household contacts

\section{Introduction}

There is mounting evidence that a new onset of altered sense of smell or taste is strongly related to severe acute respiratory syndrome coronavirus 2 (SARS-CoV-2) infection. The

Daniele Borsetto

daniele.borsetto@gmail.com

1 Section of Otorhinolaryngology, University of Padova, Treviso, Italy

2 Guy's and St Thomas' Hospitals, London SE1 9RT, UK

3 Section of Otorhinolaryngology, University of Padova, Padova, Italy

4 Head and Neck Department, Cattinara Hospital, University of Trieste, Trieste, Italy

5 Department of Clinical Pathology, AULSS 2, Marca Trevigiana, Treviso, Italy

6 Unit of Cancer Epidemiology, Aviano National Cancer Institute, IRCCS, Aviano, Italy prevalence of self-reported smell or taste impairment in patients with coronavirus disease 2019 (COVID-19) ranges from 11 to $87 \%$ in severe and mild-moderate cases, respectively [1,2]. Moreover, the objective evaluation of the sense of smell showed that self-reporting surveys may even underestimate the prevalence of anosmia in COVID-19 patients $[3,4]$.

According to World Health Organization (WHO) recommendation, patients testing positive for SARS-CoV-2 and having mild illness and no risk factors can be managed at home, as long as they can be followed up and cared for by family members [5].

In our region, following the recommendations of the European Centre for Disease Prevention and Control [6], household contacts of SARS-CoV-2-positive subjects are placed in self-isolation for 14 days with daily monitoring for COVID-19 symptoms, including fever of any grade, cough or difficulty breathing, with nasopharyngeal and throat swabs being performed only in subjects that develop these symptoms. 
However, a substantial proportion of SARS-CoV-2 infections may be asymptomatic or paucisymptomatic [7] with the sudden onset of the loss of sense of smell or taste reported as isolated symptom in 3\% of cases [8].

To better estimate the burden of smell and taste impairment during COVID-19 pandemic, we searched for the prevalence of these symptoms in subjects at high risk for SARS-CoV-2 infection, i.e. household contacts of mildly symptomatic home-isolated SARS-CoV-2-positive patients.

\section{Methods}

We previously reported the prevalence of loss of the sense of smell or taste as well as other COVID-19 symptoms in a case series of 202 home-isolated mildly symptomatic confirmed cases of SARS-CoV-2 infection [8].

All these patients and an additional 27 other subjects with PCR-positive SARS-CoV-2 infection residents in Treviso province, Italy, were contacted during March and April 2020, to record symptoms and their evolution. During telephone interviews, home-isolated patients were questioned regarding the presence of adult ( $\geq 18$ years) home-isolated household contacts. Consenting household contacts were then themselves asked about the presence of COVID-19-related symptomatology, as well as about new onset of smell or taste impairment. To reduce respondent burden, we omitted to address the intensity and the timing of the onset of smell or taste loss in respect to other symptoms.

Household contacts were asked if they had undergone nasopharyngeal and throat swabs for SARS-CoV-2 detection and the testing result. All residents in Treviso province are referred to COVID-19 referral center where, according to WHO recommendations [9], SARS-CoV-2 infection is searched for by real-time polymerase chain reaction (RTPCR) in nasopharyngeal and throat swabs.
Symptom prevalence was expressed as the percentage of total patients, and 95\% confidence interval (CI) was calculated using the Clopper-Pearson method. Prevalences were compared using Fisher exact test. $P$ values $<0.05$ (two sided) were considered statistically significant. Statistical analyses were performed using R 3.6.

The study was conducted with the approval of the institutional ethical review board and informed consent was obtained verbally for telephone interviews.

\section{Results}

Of 229 mildly symptomatic COVID-19 patients, 214 (93.4\%) were successfully contacted: two patients died after study enrollment due to a sudden and rapid deterioration of general conditions and additional 13 patients, of which two needed hospitalization, did not answer or refused the interview.

Among these 214 patients, 179 reported to have at least one household contact during their home isolation period, with the total number of study participant's contacts available for the interview being 296 .

Table 1 shows symptoms experienced by 296 household contacts of home-isolated SARS-CoV-2-positive subjects according to the swab test. Overall, $74(25.0 \%, 95 \% \mathrm{CI}$ 20.2-30.3\%) household contacts complained new onset of the loss of smell or taste.

Of these, 121 underwent nasopharyngeal and throat swabs to detect SARS-CoV-2 by RT-PCR: 54 household contacts tested positive for SARS-CoV-2 and 34 (63.0\%, 95\% CI 48.7-75.7) had loss of smell or taste, while 67 tested negative and $1(1.5 \%, 95 \%$ CI $0.0-8.0)$ had loss of smell or taste. Thus, a new onset of altered sense of smell and taste has reported a sensitivity of $63.0 \%$ (95\% CI 48.7-75.7\%) and a specificity of $98.5 \%$ (95\% CI $92.0-100 \%$ ) in detecting COVID-19-positive patients, with a positive predictive value

Table 1 Symptoms in 296 household contacts of 179 home-isolated SARS-CoV-2-positive subjects

\begin{tabular}{|c|c|c|c|c|c|c|c|c|c|c|}
\hline \multirow[t]{3}{*}{ Symptoms } & \multicolumn{9}{|c|}{ RT-PCR for SARS-CoV-2 } & \multirow[t]{3}{*}{ Fisher exact test } \\
\hline & \multicolumn{3}{|c|}{ Tested positive } & \multicolumn{3}{|c|}{ Tested negative } & \multicolumn{3}{|c|}{ Not tested } & \\
\hline & $n$ & $(\%)$ & $(95 \% \mathrm{CI})$ & $n$ & $(\%)$ & $(95 \% \mathrm{CI})$ & $n$ & $(\%)$ & $(95 \% \mathrm{CI})$ & \\
\hline Total patients & 54 & 18.3 & & 67 & 22.6 & & 175 & 59.1 & & \\
\hline Fever & 29 & 53.7 & $(39.6-67.4)$ & 12 & 17.9 & $(9.6-29.2)$ & 65 & 37.1 & $(30.0-44.8)$ & $p<0.001$ \\
\hline Dry or productive cough & 21 & 38.9 & $(25.9-53.1)$ & 10 & 14.9 & $(7.4-25.7)$ & 39 & 22.3 & $(16.4-29.2)$ & $p=0.008$ \\
\hline Problems breathing & 14 & 25.9 & $(15.0-39.7)$ & 1 & 1.5 & $(0.0-8.0)$ & 3 & 1.7 & $(0.0-4.9)$ & $p<0.001$ \\
\hline Altered sense of smell or taste & 34 & 63.0 & $(48.7-75.7)$ & 1 & 1.5 & $(0.0-8.0)$ & 39 & 22.3 & $(16.4-29.2)$ & $p<0.001$ \\
\hline Other symptoms & 21 & 38.9 & $(25.9-53.1)$ & 47 & 70.1 & $(57.7-80.7)$ & 41 & 23.4 & $(17.4-30.4)$ & $p<0.001$ \\
\hline No symptoms & 0 & 0.0 & $(0.0-6.6)$ & 4 & 6.0 & $(1.7-14.6)$ & 59 & 33.7 & $(26.8-41.2)$ & $p<0.001$ \\
\hline
\end{tabular}

$S A R S-C o V-2$ severe acute respiratory syndrome coronavirus $2, R T-P C R$ real-time polymerase chain reaction 
of $97.1 \%$ (95\% CI $85.1-100 \%)$ and a negative predictive value of $76.7 \%$ (95\% CI $66.4-85.2 \%$ ).

Among 175 household contacts not tested for SARSCoV-2 infection, 67 (38.3\%, 95\% CI 31.1-45.9\%) had SARS-CoV-2-compatible symptoms, 39 (22.3\%, 95\% CI $16.4-29.2 \%)$ had loss of smell or taste with $7(4.0 \%, 95 \%$ CI 1.6-8.1\%) having loss of smell or taste in the absence of other symptoms.

Thus, the prevalence of altered sense of smell or taste was by far lower in subjects negative to SARS-CoV-2 compared to both positives $(p<0.001)$ and non-tested cases $(p<0.001)$.

\section{Discussion}

The prevalence of smell or taste impairment in household contacts of mildly symptomatic home-isolated SARS-CoV2-positive patients was $1.5 \%, 22.3 \%$, and $63.0 \%$ in subjects tested negative, non-tested, and tested positive for SARSCoV-2 infection, respectively.

These results confirmed previously reported data showing that about two-thirds of mildly symptomatic COVID-19 patients complain of an altered sense of smell or taste [8]. This is in line with experimental evidence that the transmembrane serine protease 2 and angiotensin-converting enzyme 2 (ACE2), the two key players in the binding and entry of the SARS-CoV-2 into human cells, are highly coexpressed in olfactory sustentacular cells [10], thus making this a potential target for SARS-CoV-2 infection.

In our region, nasopharyngeal and throat swabs are indicated in household contacts of SARS-CoV-2-positive subjects that develop COVID-19-related symptoms. Unfortunately, due to the temporary lack of resources (either test swabs or transportation) or patients' impaired health, comorbidities, and preferences, not all symptomatic patients have been tested. Thus, in this study, we observed that many household contacts not-tested for SARS-CoV-2 had symptoms compatible with COVID-19 and a substantial proportion complained an altered sense of smell or taste. However, 7 subjects reported only altered sense of smell or taste.

There remains a paucity of data on the risks of household transmission - although cohabiting family members are advised to maintain social distancing as the risk of transmission is thought to be high, recent reports give secondary transmission rates of 16 [11]-30\% [12]. Our study found 54 confirmed secondary infections and 75 likely cases who were not tested, suggesting a secondary infection rate of $43 \%$.

The fact that only a few patients in the group, tested negative for SARS-CoV-2, reported the absence of symptoms depends primarily on the selection bias as the public health indications are to perform the swab in symptomatic contacts. However, in most cases (70\%), these were nonspecific symptoms that did not include fever, cough, or respiratory problems. In the United Kingdom, where only symptomatic patients are tested for SARS-CoV-2 infection, among $1,460,517$ people being tested so far, as many as $1,234,054$ $(84.5 \%)$ tested negative [13]. Conversely, the prevalence of smell or taste impairment was rare in patients tested negative, thus showing that a new onset of altered sense of smell or taste has a high positive predictive value of SARS-CoV-2 infection. This makes it a very useful marker of infection where the capacity for testing is limited.

These results must be taken cautiously, due to several study limitations: data were self-reported, based on ad hoc questions, the sample was is relatively small, and only subjects $\geq 18$ years were considered.

However, this study showed that smell or taste impairment is quite common in not-tested household contacts of mildly symptomatic home-isolated SARS-CoV-2-positive patients. This should be taken into account when estimating the burden of loss of sense of smell and taste during COVID19 pandemic. Furthermore, due to the robust association between the loss of sense of smell or taste and the SARSCoV-2 infection as well as the possible existence of a monosymptomatic form of COVID-19 characterized by isolated smell or taste impairment, the new onset of these symptoms during COVID-19 pandemic should be considered a manifestation of SARS-CoV-2 infection until proven otherwise.

Funding None.

\section{Compliance with ethical standards}

Conflict of interest The authors declare that they have no conflicts of interest.

Ethics approval The study was conducted with the approval of the ethics committee for clinical experimentation of Treviso and Belluno provinces (ethic vote: 780/CE).

Informed consent Additional informed consent was obtained from all individual participants for whom identifying information is included in this article.

\section{References}

1. Mao L, Jin H, Wang M, Hu Y, Chen S, He Q et al (2020) Neurologic manifestations of hospitalized patients with coronavirus disease 2019 in Wuhan, China. JAMA Neurol. https://doi. org/10.1001/jamaneurol.2020.1127

2. Lechien JR, Chiesa-Estomba CM, De Siati DR, Horoi M, Le Bon SD, Rodriguez A et al (2020) Olfactory and gustatory dysfunctions as a clinical presentation of mild-to-moderate forms of the coronavirus disease (COVID-19): a multicenter European study. Eur Arch Oto-Rhino-Laryngol Off J Eur Fed Oto-Rhino-Laryngol 
Soc EUFOS Affil Ger Soc Oto-Rhino-Laryngol Head Neck Surg. https://doi.org/10.1007/s00405-020-05965-1

3. Vaira LA, Deiana G, Fois AG, Pirina P, Madeddu G, De Vito A et al (2020) Objective evaluation of anosmia and ageusia in COVID-19 patients: single-center experience on 72 cases. Head Neck. https://doi.org/10.1002/hed.26204

4. Moein ST, Hashemian SMR, Mansourafshar B, Khorram-Tousi A, Tabarsi P, Doty RL (2020) Smell dysfunction: a biomarker for COVID-19. Int Forum Allergy Rhinol. https://doi.org/10.1002/ alr. 22587

5. Home care for patients with COVID-19 presenting with mild symptoms and management of their contacts n.d. https://www. who.int/publications-detail/home-care-for-patients-with-suspe cted-novel-coronavirus-(ncov)-infection-presenting-with-mildsymptoms-and-management-of-contacts. Accessed 27 Apr 2020

6. COVID-19. Eur Cent Dis Prev Control n.d. https://www.ecdc. europa.eu/en/covid-19-pandemic. Accessed 27 Apr 2020

7. Nishiura H, Kobayashi T, Suzuki A, Jung S-M, Hayashi K, Kinoshita R et al (2020) Estimation of the asymptomatic ratio of novel coronavirus infections (COVID-19). Int J Infect Dis IJID Off Publ Int Soc Infect Dis. https://doi.org/10.1016/j.ijid.2020.03.020

8. Spinato G, Fabbris C, Polesel J, Cazzador D, Borsetto D, Hopkins C et al (2020) Alterations in smell or taste in mildly symptomatic outpatients with SARS-CoV-2 infection. JAMA. https://doi. org/10.1001/jama.2020.6771
9. Technical guidance n.d. https://www.who.int/emergencies/disea ses/novel-coronavirus-2019/technical-guidance. Accessed 27 Apr 2020

10. Fodoulian L, Tuberosa J, Rossier D, Landis BN, Carleton A, Rodriguez I (2020) SARS-CoV-2 receptor and entry genes are expressed by sustentacular cells in the human olfactory neuroepithelium. BioRxiv. https://doi.org/10.1101/2020.03.31.013268

11. Li W, Zhang B, Lu J, Liu S, Chang Z, Cao P et al (2020) The characteristics of household transmission of COVID-19. Clin Infect Dis Off Publ Infect Dis Soc Am. https://doi.org/10.1093/cid/ciaa4 50

12. Wang Z, Ma W, Zheng X, Wu G, Zhang R (2020) Household transmission of SARS-CoV-2. J Infect. https://doi.org/10.1016/j. jinf.2020.03.040

13. COVID-19: track coronavirus cases. GOVUK n.d. https://www. gov.uk/government/publications/covid-19-track-coronavirus-cases . Accessed 13 May 2020

Publisher's Note Springer Nature remains neutral with regard to jurisdictional claims in published maps and institutional affiliations. 